\title{
Institutionalization of stock status report in the management of HIV/AIDS programme: experience from Nigeria
}

\author{
Ademola Joshua Itiola ${ }^{1}$, Chinedu Obi ${ }^{1}$, Abubakar Mohammed ${ }^{1}$, Joseph Raji , Innocent Ibegbunam ${ }^{1}$, Sunday Aguora ${ }^{1}$, \\ Adamu Sambo', Babatunji Odelola ${ }^{2}$ \\ 1 Supply Chain Management System, Abuja, Nigeria, 2 United States Agency for International Development, Abuja, Nigeria \\ Keywords: global health \\ https://doi.org/10.29392/joghr.3.e2019010
}

\section{Journal of Global Health Reports}

Vol. 3, 2019

\begin{abstract}
Incomplete and poor quality of data generated by routine health information systems limit their use in program planning, implementation and monitoring. Prior to 2012, there was no central structure in place in Nigeria for the collation, analysis and presentation of HIV/AIDS Logistics Management Information System (LMIS) data in a usable format. Following the resolution to unify multiple HIV/AIDS supply chains, the country embarked on an aggressive effort to improve the quantity and quality of LMIS reports. This effort culminated in the periodic generation of Stock Status Report (SSR). The report ensures that LMIS data are collated, analysed and presented in a format that enables stakeholders make informed decisions. In the course of implementation, the country also simplified LMIS tool and adopted a mix of report collection models. The SSR has ensured that Nigeria has a repository of LMIS data that aids program planning, implementation and monitoring. In addition, report generation has stimulated improvement in LMIS data quality for HIV/AIDS and other public health programmes. Our experience suggests that data from routine health information systems (RHIS) is of value in public health programming in resource limited settings. Positive spillover effect is also feasible while simplification of data collection tools can indeed help improve quantity and quality of reports generated through RHIS.
\end{abstract}

Health information system is an integral part of a strong health system given the critical role information plays in programmme planning, implementation, monitoring and evaluation. ${ }^{1}$ One of the sources of health information is routine health services data; when these data are available and of good quality, they are useful for identification of service gaps which inform institution of service improvement measures. ${ }^{2}$ The major drawbacks that however limit the use of routine service data are their non-availability and poor quality. ${ }^{2}$ Available evidence also suggests that routine service data are collected as an end in themselves rather than means for driving actions that improve the health system. ${ }^{3-5}$ Reasons that have been established for non-availability or poor quality of routine data include: inadequate and poorly motivated staff, non-availability of data collection tools and infrastructural deficits to mention but a few. ${ }^{4}$

These challenges underscore the need to put in place structures that guarantee periodic collection, validation, collation, analysis and use of routine data for action.

\section{SETTINGS}

Nigeria's population is estimated to be approximately 191 million as of 2017 with HIV prevalence of $2.9 \% .^{6,7}$ HIV response in the country is largely funded by President's Emergency Fund for AIDS Relief (PEPFAR), Global Fund and the World Bank. ${ }^{8,9}$ The HIV/AIDS supply chain system is a 2-tier system; health facilities send their logistics manage- ment information system (LMIS) reports in form of a combined report requisition issue and receipt form (CRRIRF) to the central level on a bimonthly basis and are resupplied directly from six central warehouses (zonal hubs) to preset maximum stock level. ${ }^{10,11}$ This CRRIRF report summarizes consumption, quantity of stock received within the review period, and stock on hand amongst other data elements. Prior to July 2012, individual implementing partners resupply their supported health facilities through their separate supply chain systems. With Unification, all the supply chain systems were unified into one by July $2014^{12}$ - see Itiola \& Agu, 2018 for a more comprehensive description of the HIV/ AIDS Supply Chain System. ${ }^{13}$

\section{PROBLEM STATEMENT}

Prior to August 2012, there was no structure in place at the national level that collates, analyses and presents LMIS data in a format that is usable for decision making partly due to the non-unified supply chain systems. This limited the availability of logistics data for informed decisions.

\section{INTERVENTION}

\section{APPROACH}

Following the resolution to unify multiple HIV/AIDS supply chains in Nigeria by the Procurement and Supply Manage- 
ment Technical Working Group (PSM TWG), the country embarked on an aggressive effort to improve the quantity and quality of LMIS reports in 2012. This effort culminated in the generation of Bimonthly National Stock Status Report. This effort was led by PEPFAR-funded Supply Chain Management System (SCMS) project with oversight from PSM TWG.

\section{THE REPORT: STOCK STATUS REPORT (SSR)}

The stock status report (SSR) provides information on available in-country stock at the end of a review period across all storage points, how long these stocks will last (expressed in months), consumption pattern, shipment information, information on short-dated commodities (6 months or less for drugs and 3 months or less for laboratory commodities) and redistributed commodities. Recommendations are also proffered to address any observed stock imbalance. In addition, the report provides updates on programmatic issues that have implications for commodity use. Case in point was regimen streamlining - reduction of the number of antiretroviral drugs products and opportunistic infection medicines from over 50 to 21 .

\section{METHODOLOGY FOR REPORT GENERATION}

To generate the stock status report, CRRIRF and stock reports from all health facilities and warehouses are collected and reviewed. Following reviews, information on stock on hand from all warehouses and health facilities are aggregated to get the overall stock on hand for the country; consumption across all health facilities in the country is also aggregated. Months of stock (MOS) is then estimated by dividing aggregate stock on hand by aggregate average monthly consumption. Deductions are then made as to whether or not the country is optimally stocked based on acceptable minimum and maximum inventory control levels. The report narrative also provides contextual explanation for observed MOS. In addition, central level stock on hand is reviewed to establish if there are short-dated commodities. Finally updates on programmatic issues are presented. Recently MOS has been modified to MOS cover; in the latter stock that will potentially expire (based on projected consumption trend) is discounted from the stock on hand.

\section{PROGRESSION OF THE STOCK STATUS REPORT}

In August 2012 when stock status report generation started, it was only generated at the national level and covers just antiretroviral drugs and cotrimoxazole partly due to the fact that these were the only pool procured HIV/AIDS commodities as of then. With addition of more commodities to the pool procurement structure, other commodities were included in the report (HIV rapid test kits - March/April 2013; CD4 reagents - May/June 2013; Polymerase Chain Reaction (PCR) commodities - March/April 2015).

With time, the need to have individual state level report was identified as this is critical to promoting state ownership and use of LMIS data for action. These state reports also help unearth details that may have been masked in the course of data aggregation at the national level. This led to the institution of state-level stock status report which was not limited to HIV/AIDS commodities but also extended to other public health commodities (malaria, tuberculosis, family planning and vaccines) - integrated stock status report - (ISSR). To operationalize this, a standard operating procedure which guided mentorship of states was developed. The integrated stock status report is now generated on a quarterly basis and coordinated by the Logistics Management Coordinating Unit (LMCU), Nigeria Supply Chain Integration Project (NSCIP) with support from Global Health Supply Chain Procurement and Supply Management (GHSC-PSM) project and other implementing partners.

As of the end of July/August 2018 review period, a total of 35 editions of National SSR had been produced. NSCIP has equally led the generation of five editions of the Quarterly Integrated National Stock Status report.

\section{POST INTERVENTION ASSESMENT}

In assessing the impact of this intervention, we conducted desk review of programmatic reports and auto reflected on the changes that had happened since the introduction of the stock status report. Detailed below are the benefits the country has derived since the introduction.

\section{BENEFITS}

\section{REPOSITORY OF LOGISTICS DATA}

With the stock status report, Nigeria can boast of HIV/AIDS LMIS data repository and reference documents from which historical information on stock and shipment management can be accessed. This includes information on: in-country consumption which gives an idea of the average spending on HIV commodities and can ultimately aid planning for sustainable funding as well as retrieval/redistribution activities carried out to salvage expiries - $\$ 190,847$ worth of products were retrieved between August and October 2013. 14 Review of the bimonthly national stock status report for the review period extending from November 2013 - August 2015 revealed that an additional \$1,271,486 worth of products were either retrieved or redistributed.

\section{IMPROVED LMIS DATA QUALITY}

In the course of review of stock and CRRIRF reports, facilities with data quality issues are identified. Any quality issue that may have been missed out are also identified following aggregation - anomalous data (consumption/stock on hand) trend is an indication for further review. The concerned focal persons are then contacted on rectification of observed error(s) and also mentored on proper reporting.

\section{IMPROVED LMIS DATA MANAGEMENT FOR OTHER PUBLIC HEALTH PROGRAMME}

The report has had catalytic impact in stimulating other public health programmes to work towards strengthening collection of facility-level LMIS data. Integrated stock status report (with information on other public health pro- 
grammes) is now generated both at the national and state levels.

\section{PROGRESS MONITORING}

The stock status report allows for consistent monitoring of progress of key programmatic changes. A major programmatic change that occurred following 2013 national quantification exercise was regimen streamlining. The stock status report assisted in monitoring the progress of this exercise while ensuring the non-adherent health facilities are identified and actively followed up for compliance.

\section{VALUABLE SOURCE OF DATA FOR DEMAND/SUPPLY PLANNING AND FORECASTING ASSUMPTIONS}

The report had ensured consistent availability of data (consumption and stock on hand) for updating the national commodity pipeline which aids both supply and demand planning. It has also guaranteed availability of key assumptions for forecasting exercise. Case in point is the availability of utilization-based positivity and discordant rates (5\% and 1\% respectively) for HIV rapid test kits forecasting. Similarly, historical consumption trend serves as valuable source of information for validating forecast output and establishing forecast accuracy (and subsequent forecast revision where indicated). This ultimately helps prevent overstock and stock out.

\section{CHALLENGES}

The main challenges with report generation are related to late reporting and time-consuming nature of data aggregation. At the inception of stock status report, LMIS reports were manually entered into an access database (and later PHP runner) before the final analysis. Given the huge number of health facilities providing HIV/AIDS services in Nigeria (>7,000 as of 2015), 15 collection and aggregation of CRRIRF reports is tedious and time consuming. Instances exist where some health facilities report late or transmit poor quality report that require further follow up. Also, industrial strike actions sometimes limit the volume of reports available for decision making.

\section{MITIGATION STRATEGIES}

Strategies adopted to address these challenges include: active follow up with health facilities to facilitate timely transmission of reports, leveraging existing relationship with relevant staff to facilitate report submission during industrial strike action, review of CRRIRF reports and engagement of relevant staff to address observed data quality issue. To ensure sustained reporting rate with rapid scale up of HIV/AIDS services, the country also simplified CRIRFF for health facilities providing prevention of mother to child transmission of HIV (PMTCT) and HIV testing and counselling (HTC) services - the simplified tool had the endorsement of all stakeholders (obtained through the PSM TWG) after which it was deployed to health facilities. To operationalize the use of the tool, health facility staff were men- tored during national monitoring and supportive visits and other supervisory visits by state government staff and implementing partners. The country also adopted a mix of report collection models - Cluster review meetings, Use of Local Government Area (LGA) Officers, Hub and Spoke Model and email submission). With this mix of interventions, reporting rate increased from $70 \%(2,744 \mathrm{HFs})$ in July 2013 to $87 \%$ (7,389 HFs) in March 2015). ${ }^{15}$ In order to reduce the time required for data aggregation, the country is currently scaling up electronic LMIS which will totally eliminate the phase of manual entry of data into the access database.

Overall the LMIS reporting rates for HIV/AIDS programme across all states in Nigeria has been largely good over the course of report generation (over $80 \%$ in most instances) thereby making deductions from MOS estimates as well as other indicators representative. We however anticipate that this may not necessarily be the case in other countries that may want to adopt this intervention. While countries are encouraged to work on the underlying causes of poor reporting such as complexity of data collection tool and lack of clear report collection structure, an approach we would recommend is for countries to adjust incomplete data to estimate complete reporting as this will ensure the representativeness of deductions. A more detailed description of this approach can be found in The Logistics Handbook: A Practical Guide for the Supply Chain Management of Health Commodities. ${ }^{11}$

\section{LESSONS LEARNED}

Strong coordination structure as well as skilled and committed human resource is required for prompt report generation: The solid oversight provided by the leadership in SCMS project as well as PSM TWG ensured continuous generation of the report. This includes periodic demand for evidence (from stock data analysis) before programmatic decisions are made.

Clear report collection mechanism is critical for prompt report collection: The delegation of report collection role to a single organization with unification project guaranteed strong central coordination for report collection which is a critical process for harvesting data required for report generation. The use of mix of report collection models based on individual state's peculiarity also facilitated prompt report collection.

Simplification of LMIS tool can improve quality and quantity of LMIS data: In response to the need to have a tool that can be used by health facilities providing PMTCT and HTC services who may not have as much specialized manpower as those providing comprehensive antiretroviral therapy services, the country simplified the CRIRRF for PMTCT and HTC sites. This ensured that the country is able to obtain quality reports from this category of health facilities.

Use of routine data drives improvement in quality: Regular review of LMIS data ensured prompt identification of data quality issues and their resolution. This validates the assertion that regular review and use of routine data can drive improvement in quality.

Positive spillover effect: The stock status report which started with HIV/AIDS commodities has now been extended 
to other public health programs; this suggests that best practices in a particular public health program are transferrable and can have beneficial impact on other public health programs.

\section{CONCLUSION}

Our experience suggests that routine data is of value in program planning, implementation and monitoring in resource limited settings even when all processes are not fully automated. Positive spillover effect is also feasible while simplification of data collection tools can indeed help improve quantity and quality of reports generated through RHIS.

Cooperation of all stakeholders within the public health space is required in sustaining the gains that have been achieved while institution of electronic LMIS will ensure more timely report generation as this will eliminate the phase of manual entry of reports prior to analysis. Automation will also facilitate easy triangulation of logistics and program data. Other countries may want to explore generation of similar report as a means of driving the use of LMIS data for action.

\section{ACKNOWLEDGEMENTS}

Supply Chain Management System (SCMS) project under which generation of stock status report was initiated was funded by PEPFAR. We also appreciate the effort of healthcare workers in documenting and reporting the data used in developing stock status report. The opinions expressed in this paper are strictly those of the authors and do not necessarily reflect the views of the institution of affiliation of the authors.

\section{FUNDING}

None.

\section{COMPETING INTERESTS}

The authors completed the Unified Competing Interest form at http://www.icmje.org/coi_disclosure.pdf (available upon request from the corresponding author), and declare no conflicts of interest.

\section{CORRESPONDENCE TO:}

Ademola J. Itiola

P.O Box 2586

Dugbe

Ibadan

Oyo State

Nigeria

ademola.itiola@gmail.com 


\section{REFERENCES}

1. World Health Organization. Everybody's business: Strengthening health systems to improve health outcomes. WHO's framework for action. Published 2007. Accessed January 4, 2018. http://www.who.int/h ealthsystems/strategy/everybodys business.pdf

2. Braa J, Heywood A, Sahay S. Improving quality and use of data through data-use workshops: Zanzibar, United Republic of Tanzania. Bull World Health Organ. 2012;90(5):379-384. doi:10.2471/blt.11.099580

3. Aqil A, Lippeveld T, Hozumi D. PRISM framework: a paradigm shift for designing, strengthening and evaluating routine health information systems. Health Policy Plan. 2009;24(3):217-228. doi:10.1093/h eapol/czp010

4. Sambo MN, Lewis I, Sabitu K. Quality of record system in primary health centres of Tafa LGA, North Central Nigeria. Ann Niger Med. 2005;1:15-18.

5. Opit JL. How should information on healthcare be generated and used? Round Table and discussion. World Health Forum. 1987;8:409-438.

6. The World Bank Group. Poverty \& Equity Data Portal, Nigeria. Published 2018. Accessed June 10, 2018. http://povertydata.worldbank.org/poverty/coun try/NGA

7. National Agency for the Control of AIDS. National HIV and AIDS Strategic Plan 2017- 2021. Published 2017. Accessed April 24, 2018. https://naca.gov.ng/w p-content/uploads/2018/05/National-HIV-and-AIDSStrategic-Plan-FINAL1.pdf

8. Ezegbe C, Stephenson N. The reach and limits of the US president's emergency plan for aids relief (PEPFAR) funding of prevention of mother-to-child transmission (PMTCT) of HIV in Nigeria. Afr J Reprod Health. 2012;16:23-34.
9. Ibegbunam I, McGill D. Health Commodities Management System: Priorities and Challenges. Journal of Humanitarian Logistics and Supply Chain Management. 2012;2(2):161-182. doi:10.1108/204267 $\underline{41211260741}$

10. Federal Ministry of Health. Logistics Management of HIV/AIDS Commodities, Standard Operating Procedures Manual for the Management of HIV/AIDS Commodities, Antiretroviral Drugs, OI Drugs, Laboratory Reagents and Supplies.; 2011.

11. USAID / DELIVER Project. The Logistics Handbook: A Practical Guide for the Supply Chain Management of Health Commodities. USAID / DELIVER Project, Task Order $1 ; 2011$.

12. Itiola A, Obi C, Raji J, et al. Government Partnership and Private Sector Engagement: Drivers for optimal performance of Nigeria HIV/AIDS Supply Chain System. Published online 2014.

13. Itiola AJ, Agu KA. Country ownership and sustainability of Nigeria's HIV/AIDS Supply Chain System: qualitative perceptions of progress, challenges and prospects. J Pharm Policy Pract. 2018;11:21. doi:10.1186/s40545-018-0148-8

14. Itiola A, Egharevba M, Ibegbunam I, Adedoyin D, Iyeme E, Obi C, et al. Ensuring Anti-retroviral Availability in a Transitional Phase: The Nigerian Experience. Published 2014. Accessed December 24, 2018. http://pag.aids2014.org/abstracts.aspx?aid=403 7

15. Itiola A, Mohammed A, Attah M, Stephen A, Haruna J, Iwheye-Adie B, et al. Simplification of logistics tools and mixed report collection models: Agents of HIV/AIDS commodity security in the era of rapid program scale up in Nigeria. Published online 2015. 